\title{
A prognostic model to predict the success of artificial insemination in dairy cows based on readily available data
}

\author{
C. J. Rutten, ${ }^{* 1}$ W. Steeneveld, ${ }^{*} \dagger$ J. C. M. Vernooij, ${ }^{*}$ K. Huijps, $\neq^{2}$ M. Nielen, ${ }^{*}$ and H. Hogeveen ${ }^{*} \dagger$ \\ *Department of Farm Animal Health, Faculty of Veterinary Medicine, Utrecht University, 3584 CL, Utrecht, the Netherlands \\ †Business Economics Group, Wageningen University, $6706 \mathrm{KN}$, Wageningen, the Netherlands \\ fCRV, 6800 AL Arnhem, the Netherlands
}

\begin{abstract}
A prognosis of the likelihood of insemination success is valuable information for the decision to start inseminating a cow. This decision is important for the reproduction management of dairy farms. The aim of this study was to develop a prognostic model for the likelihood of successful first insemination. The parameters considered for the model are readily available on farm at the time a farmer makes breeding decisions. In the first step, variables are selected for the prognostic model that have prognostic value for the likelihood of a successful first insemination. In the second step, farm effects on the likelihood of a successful insemination are quantified and the prognostic model is cross-validated. Logistic regression with a random effect for farm was used to develop the prognostic model. Insemination and test-day milk production data from 2,000 commercial Dutch dairy farms were obtained, and 190,541 first inseminations from this data set were used for model selection. The following variables were used in the selection process: parity, days in milk, days to peak production, production level relative to herd mates, milk yield, breed of the cow, insemination season and calving season, $\log$ of the ratio of fat to protein content, and body condition score at insemination. Variables were selected in a forward selection and backward elimination, based on the Akaike information criterion. The variables that contributed most to the model were random farm effect, relative production factor, and milk yield at insemination. The parameters were estimated in a bootstrap analysis and a cross-validation was conducted within this bootstrap analysis. The parameter estimates for body condition score at insemination varied most, indicating that this effect varied most among Dutch dairy farms. The cross-validation showed that
\end{abstract}

Received January 22, 2016.

Accepted April 14, 2016.

${ }^{1}$ Corresponding author: c.j.rutten@uu.nl

${ }^{2}$ Current address: kirstenvanhees.nl, 5451 NR Mill, the Netherlands. the prognosis of insemination success closely resembled the mean insemination success observed in the data set. Insemination success depends on physiological conditions of the cow, which are approximated indirectly by production and reproduction data that are routinely recorded on the farm. The model cannot be used as a detection model to distinguish cows that conceive from cows that do not. The model validation indicates, however, that routinely collected farm data and testday milk yield records have value for the prognosis of insemination success in dairy cows.

Key words: dairy, prognostic model, insemination success, reproduction

\section{INTRODUCTION}

Reproduction management has a large influence on dairy farm profitability (Inchaisri et al., 2010b; Giordano et al., 2011). Economically important factors include the decision to start inseminating, the voluntary waiting period (VWP; Inchaisri et al., 2011b), and the decision to stop inseminating a dairy cow (Inchaisri et al., 2012). The goal underlying the choice of VWP is to realize a defined calving interval and milk yield at dry off, which requires a cow to conceive before a certain DIM. The optimal VWP within a lactation depends on factors such as daily milk yield, production level, estrus detection rate, and the cow's health status (Inchaisri et al., 2011b). A prognosis of the likelihood of insemination success is valuable information for the decision to start with insemination, and can therefore improve reproduction management.

Although one study focused on prognosis of the likelihood of insemination success (Shahinfar et al., 2014), most studies have focused on identifying risk factors associated with insemination success (Caraviello et al., 2006; Gábor et al., 2008; Inchaisri et al., 2010a, 2011a; Lane et al., 2013; Löf et al., 2014). These studies have identified the following risk factors: milk yield, parity, cow breed, insemination number, lactation stage, calving season, insemination season, use of sexed semen, and information on the course of the previous calving 
(e.g., whether dystocia occurred) for insemination success.

Other factors thought to influence insemination success in dairy cows include diseases (Fourichon et al., 2000; Canu et al., 2010), BCS, ketosis, SCC, and breed of the cow (Caraviello et al., 2006; Lane et al., 2013; Löf et al., 2014). An important factor is BCS, which can be seen as a proxy of the dairy cow's body reserves (Löf et al., 2014). In particular, a postpartum decrease in BCS, indicating mobilization of body reserves, is associated with lower reproductive performance (Roche et al., 2009; Hernandez et al., 2012). In case of extreme mobilization of body reserves, ketosis may occur, which is also associated with lower reproductive performance (Roche et al., 2009; Schefers et al., 2010). Ketosis could be included in an analysis of the likelihood of insemination success by using a ketosis score from milk recordings, which is based on ketone bodies in milk (de Roos et al., 2007), or on the ratio of fat to protein content. Many variables with potential prognostic value for the likelihood of successful insemination, such as fat and protein contents, SCC, lactose content, and ketosis score, have been evaluated as risk factors for insemination success.

Several studies have shown the importance of farm factors for the reproductive performance of dairy herds (Schefers et al., 2010; Shahinfar et al., 2014). For insemination success in individual cows, herd conception rate in the past 3 mo has been suggested as an important predictor (Shahinfar et al., 2014). Other farm factors found to be influential are VWP, estrus detection accuracy, stocking density, udder health level of the herd, and use of a resynchronization program (Schefers et al., 2010). It is important, therefore, that models which give a prognosis of insemination success account for farm effects and quantify these effects.

It is logical to assume that risk factors having a strong association with insemination success are useful for making a prognosis. However, the strength and causality of association give no indication of the prognostic power of a parameter. To implement a prognosis of the likelihood of insemination success in a decision support system, it is important to understand the accuracy of the prognosis. This requires knowledge of the robustness of the estimated parameters in a prognostic model, given the variation among farms and individual cows. Furthermore, it is important to conduct an independent validation of the prognosis. Shahinfar et al. (2014) conducted a validation for machine learning algorithms and reported an area under the receiver operating characteristic curve (AUC) in the range of 0.6 to 0.75 .

The aim of this study was to develop a prognostic model for the likelihood of successful first insemination. In the first step, variables were selected for the prognostic model that had prognostic value for the likelihood of a successful first insemination. The selected variables are readily available on-farm when a farmer makes breeding decisions and are obtained from data that is currently routinely recorded on Dutch dairy farms. In the second step, the farm effects on the likelihood of a successful insemination were quantified and the prognostic model was cross-validated.

\section{MATERIALS AND METHODS}

\section{Available Data}

Data on inseminations, calving dates, parity and age of the cow, pregnancy checks, lactation length, relative production factor (a factor that represents the production level of an individual cow relative to her herd mates), and test-day milk yields from 2,000 randomly selected dairy farms, covering the period 2009 to 2012, were obtained from the database of the Dutch cattle breeding and milk production recording company (CRV, Arnhem, the Netherlands). In 2009 and 2012, 20,268 and 18,682 farms participated in the milk production recording program of CRV, representing 81 and $85 \%$ of the total number of farms, respectively. Selected farms were required to have a farm size of at least 50 cows and to make use of the CRV AI service. Records were removed from the data set when the data were considered biologically implausible. For example, one test-day was removed as the lactose percentage was $15 \%$.

The data set contained 1,403,194 recorded AI and 613,668 individual lactations. Only records containing inseminations with a known preceding calving date (so the calving date that started the lactation in which the cow was inseminated at a certain DIM) were selected; 653,694 recorded AI were removed from the initial data set because no preceding calving date was registered, leaving 749,500 records. The focus in this study was on the first insemination of a lactation. A farmer will choose to start breeding a cow after a certain DIM. If insemination is unsuccessful, the farmer decides either to inseminate the cow again or to stop inseminating and cull, rather than choosing to postpone a reinsemination. A prognosis of the likelihood of insemination success is therefore most relevant for the first insemination of a lactation.

\section{Definition of Successful and Unsuccessful First Inseminations}

Inseminations recorded in the database were considered unsuccessful when, within the current lactation, another insemination occurred within $100 \mathrm{~d}$ after the 
date of first insemination. An insemination was considered successful when a calving was registered in the period from 250 to $310 \mathrm{~d}$ after insemination, without a subsequent insemination. This definition considered as unsuccessful those inseminations that led to conception but did not result in calving. If neither a calving nor a subsequent insemination was registered after an insemination, the pregnancy status derived from the pregnancy check was used to determine insemination success. The pregnancy check was considered to correspond with the first insemination if it occurred between 20 and $100 \mathrm{~d}$ after insemination. If neither a calving nor a subsequent insemination nor a pregnancy check was registered after a first insemination, then this insemination was removed from the data set. This procedure resulted in a data set containing 365,128 first inseminations.

\section{Fitting Lactation Curves}

Test-day milk recordings were available for 552,235 lactations. We assumed that farmers using this prognostic model would either have milk meters in the milking parlor or an automated milking system to record daily milk yields for each cow. However, data on daily milk yield were not available in the data set used for model development. In addition, test-day milk yields did not necessarily correspond with the day of insemination. The daily milk yields were therefore interpolated based on lactation curves. Lactation curves were estimated to determine milk yield and characteristics of the lactation curve corresponding to the day of insemination. A nonlinear least squares estimation with the LevenbergMarquardt algorithm was used to fit lactation curves for individual lactations of dairy cows. The LevenbergMarquardt algorithm is robust in reaching convergence and produces small standard errors compared with other algorithms (Dematawewa et al., 2007).

Milk. Using test-day results from the first 250 farms in the data set, group models were estimated for the following curves: Wood (Wood, 1967), Wilmink (Wilmink, 1987), Dijkstra (Dijkstra et al., 1997), and Rook (Rook et al., 1993). Starting values were obtained from the 305-d milk yield models of Dematawewa et al. (2007). The Bayesian information criterion (BIC) was used to compare the group models based on parsimony and fit. A more parsimonious model was preferred, because individual production curves are fitted on a limited number of test-day recordings. The BIC places more emphasis on parsimony by using a higher penalty for an extra variable added to the model compared with other criteria, such as the Akaike information criterion (AIC).
The Dijkstra curve (Equation [1]) had the lowest BIC for milk yield. Previous comparisons of production curves indicated that the Dijkstra curve provided the most accurate estimates of peak milk yield and time of peak milk yield and that smaller models are preferable (Val-Arreola et al., 2004; Dematawewa et al., 2007). The Dijkstra curve was chosen as the production curve to model milk yield based on the BIC in the current study and previous findings. The estimated model coefficients $(a, b, c$, and $d$, as well as Euler's number, $e$ ) from the group model were used as starting values for fitting individual production curves. These starting values are presented in Table 1;

$$
\text { Milk yield }(\mathrm{kg} / \mathrm{d})=a \times e^{\frac{b \times\left(1-e^{-c \times D I M}\right)}{c}-d \times D I M} .
$$

Selection criteria for the data were designed to fit individual production curves. First, the test-day milk yield was required to be $<80 \mathrm{~kg}$, as higher values were considered biologically implausible. Second, the lactation needed to include at least 5 test-day results. Third, at least one test-day result within the first 60 DIM was required. It was assumed that in long lactations, problems such as spontaneous abortion have occurred. Therefore, lactations > 600 DIM were excluded from the analysis. The selection criteria were met by 440,140 lactations; a lactation curve was fitted for each lactation that met the selection criteria. Model coefficients for a Dijkstra curve could not be estimated for 39,466 lactations, which resulted in a data set containing 400,674 lactation curves. The maximum number of iterations for the regression analysis was set at 500 because convergence was reached for all selected lactations with this number of iterations. Milk yield curves were classified as aberrant when the coefficient $d$ was lower than or equal to 0 and coefficient $b$ was smaller than coefficient $d$, to ensure that the curve resembled the typical convex parabola production curve, similar to the approach of Inchaisri et al. (2010a). In this procedure, 44,538 curves were considered aberrant and therefore removed from the data set. The procedure of fitting production curves resulted in a Dijkstra curve for milk yield for 356,136 lactations.

Fat and Protein Contents. The fat and protein contents of the milk of an individual cow are only known on dairy farms from the results of the last testday. Therefore, the current study used the results of the last test-day before insemination for fat and protein contents. However, there is a delay between the moment of insemination and the last milk recording, and fat and protein contents can change during this period. To approximate the effect of this delay on the 
prognostic model, fat and protein contents from the milk recordings in the data set were compared with interpolated fat and protein contents. Production curves were used to interpolate fat and protein contents, following the same procedure used to fit individual milk curves. However, about half of the fat curves could not be fitted using this procedure.

Previous research has shown that the persistency of fat and protein contents differs between heifers and older cows (Dematawewa et al., 2007). Therefore, a new approach was designed to fit fat and protein curves. Separate curves were estimated for first-, second-, and third- or higher-parity cows. The Wood model (Equation [2]; Wood, 1967) was chosen, because it is a more parsimonious model and starting values for these parity groups were available (Dematawewa et al., 2007). With these starting values, group models per parity were estimated for each group of 250 farms. The coefficients of the group models were used as starting values to estimate individual lactation curves; different starting values were used for each parity. This procedure resulted in Wood production curves for 438,337 lactations for protein content, and Wood production curves for 433,029 lactations for fat content:

$$
\text { Milk yield }(\mathrm{kg} / \mathrm{d})=a+b \times D I M+c \times e^{-d \times D I M} \text {. }
$$

\section{Data Editing}

Milk production and insemination data were obtained as separate files and combined in a single file for further analysis. After combining the insemination records with the estimated production curves (curves for milk, fat, and protein), 279,785 first inseminations remained in the data set. The number of first inseminations decreased because at least one of the production curves was not fitted appropriately for 86,343 first inseminations. The estimated production curves were used, following the methodology of Dijkstra et al. (1997), to estimate peak milk yield, day of peak milk yield, and milk yield at insemination. The fitted production curves for fat and protein were used to estimate the fat and protein contents at insemination. Subsequently, the $\log$ of the ratio of fat to protein content at insemination was calculated.

For SCC, urea, lactose percentage, and ketosis score, the last recorded test-day before insemination was used as the estimate for these parameters at insemination. Neither SCC nor urea was distributed normally and therefore their log transformations were used for further analysis.

For each insemination, the parity of the cow and BCS (score on a 5-point scale) at insemination were available. Furthermore, class variables were determined for hair color of the cow, calving season, and insemination season. Hair color of the cow contained 3 classes: black and white, red and white, and other. The season variables contained 4 classes: spring (March-May), summer (June-August), autumn (September-November), and winter (December-February).

Records that contained one or more missing values for the independent variables were removed, which resulted in a final data set containing 190,541 first inseminations (first data set) from 1,475 farms. Ketosis score and urea are optional in the production recording program and therefore not conducted on all farms. Furthermore, ketosis score and lactose percentage were only available for the most recent years of the period covered by the data set. The variable SCC also contained many missing values. Therefore, a second data set was created that included SCC, urea, lactose percentage, and ketosis score. The second data set contained 59,526 first inseminations from 1,473 farms.

Table 1. Starting values for the Levenberg-Marquardt to fit individual production curves for milk yield, fat content, and protein content; curves for fat and protein used different starting values dependent on the parity of the cow ${ }^{1}$

\begin{tabular}{lllcccc}
\hline Type & Curve & Parity & $a$ & $b$ & $c$ & $d$ \\
\hline Milk & Dijkstra $^{2}$ & All & 23.41 & 0.03557 & 0.07607 & 0.002152 \\
Fat & Wood $^{3}$ & 1 & 0.7131 & 0.1743 & 0.00183 & \\
& Wood $^{3}$ & 2 & 1.0558 & 0.1456 & 0.00283 & \\
\multirow{3}{*}{ Protein } & Wood $^{3}$ & $3+$ & 1.0503 & 0.1728 & 0.00329 & \\
& Wood $^{3}$ & 1 & 0.5209 & 0.1818 & 0.0019 & \\
& Wood $^{3}$ & 2 & 0.8257 & 0.1464 & 0.00283 & \\
& Wood $^{3}$ & $3+$ & 0.7918 & 0.1724 & 0.00329 & \\
\hline
\end{tabular}

${ }^{1} a, b, c$, and $d$ are model coefficients.

${ }^{2}$ Dijkstra et al. (1997).

${ }^{3}$ Wood (1967). 


\section{Statistical Analysis}

All data editing and analyses were conducted in $\mathrm{R}$ 3.0.2 (R Development Core Team, 2008), extended with the following packages: dPlyr (Wickham and Francois, 2014), data.table (Dowle et al., 2013), lme4 (Bates et al., 2015), reshape2 (Wickham, 2007), and minpack.lm (Elzhov et al., 2013).

Regression Model. Before the first statistical analysis was conducted, a correlation matrix was drawn up for all independent variables. Multicollinearity was assumed possible for correlations $>0.75$; in situations with possible multicollinearity, only the most biologically plausible variable was included. Possible multicollinearity was found between fat percentage at insemination and the ratio of fat to protein; only the ratio of fat to protein was used in further analyses.

The potential prognostic value of the available explanatory variables was assessed, based on univariable analyses (data not shown) and previous studies. The order of the explanatory variables in the forward selection was designed according to 2 factors: potential prognostic value and ease of use; that is, how easily the explanatory variables can be obtained and integrated in management software on dairy farms. The order was parity, DIM, log of the ratio of fat to protein content, days to peak production, relative production value, BCS at insemination, milk yield, breed of the cow, insemination season, and calving season. The independent variables were included in this order in a forward selection, using mixed logistic regression with a random intercept for farm effect (statistical analysis 1). The dependent variable was success of first insemination. The AIC was estimated in each step of the forward selection and used to balance model fit and parsimony. Model fit was considered more important than parsimony to select the best prognostic model. The data set was considered large enough to focus less on parsimony, and therefore the AIC was chosen rather than the BIC. If the AIC decreased, then the respective independent variable was kept in the model in the next step.

For the model that resulted from the forward selection, a backward elimination was conducted. In the backward elimination, the partial contribution of each independent variable to the complete model was estimated as the difference in AIC value between the full model and the model with one variable omitted. The difference in AIC indicates the contribution of an individual independent variable to the fit of the model.

In the mixed logistic regression, the maximum likelihood was approximated by a computationally demanding algorithm. The use of variables with very different scales increases computational demand, which can cause convergence problems and result in biased parameter estimates. Three variables were rescaled to prevent these problems. The variables DIM and days to peak milk yield were rescaled by dividing all values by 100. Milk yield at insemination was rescaled by dividing all values by 10 .

Bootstrap. A bootstrap procedure was applied to the model that resulted from the forward selection procedures of statistical analysis 1 to calculate confidence intervals for the estimated coefficients and estimate the bias (statistical analysis 2). For this procedure, 100 bootstrap iterations were conducted. In each iteration, farms were randomly sampled from the original data set to produce a training data set (two-thirds of the number of farms) and a test data set (one-third of the number of farms). The mixed logistic model was fitted on each training data set and the estimated coefficients were subsequently stored. Each testing data set was used for an independent validation of the model. Similar to the methodology of van der Drift et al. (2012), the models were fitted with a random herd effect to correct for clustering of cows within a farm. During validation, only the fitted values for the fixed effects were used to estimate a prognosis as the random effects would be unknown.

The same procedure of forward selection was repeated on the second data set (statistical analysis 3), containing all the variables of statistical analysis 1 and the $\log$ of SCC, log of urea, lactose percentage, and ketosis score. The contribution of each independent variable to the model fit was again assessed according to the difference in AIC in a backward elimination. After the forward selection, the bootstrap procedure was repeated for this second data set (statistical analysis 4).

Validation. In the bootstrap procedure, a prognosis for insemination success was estimated for each individual cow for each testing data set in each bootstrap iteration. Bootstrapping is a powerful validation tool for prognostic logit models (Steyerberg et al., 2001; Grobbee and Hoes, 2015). The true likelihood of insemination success was unknown so prognoses could only be validated against insemination success. Insemination success was a binary variable (successful insemination or not), whereas prognosis was a probability on a scale of 0 to $100 \%$. We assumed that an accurate prognosis would, on average, approach the mean insemination success of similar cows. Therefore, the prognoses of insemination success were summarized for each group of cows. Groups of cows with similar characteristics were defined based on the categorical variables of the data set: parity, BCS at insemination, hair color of the cow, insemination season, and calving season. The average likelihood of insemination success and average insemination success were estimated for each group of cows. The mean likelihood of insemination success and mean 
Table 2. Descriptive statistics of the available data $(\mathrm{n}=190,541)$ on insemination and production

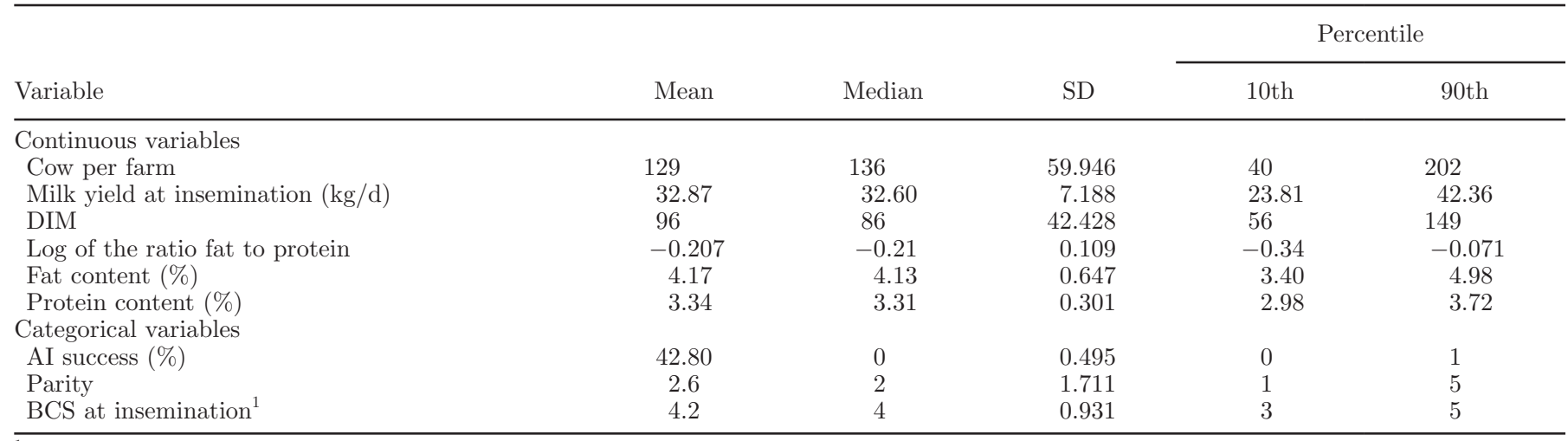

${ }^{1}$ The BCS scale contains 9 levels, ranging from 1 to 5 in increments of 0.5.

insemination success were tested for equality using a paired Wilcoxon signed rank test. The Wilcoxon signed rank test was chosen because it is appropriate for comparing the means of 2 groups when the data are not normally distributed. The test was repeated for groups of at least 100 cows to check whether the inclusion of small groups of cows influenced the difference between the mean prognosis and the mean insemination success. In some groups, all the cows became pregnant or none of the cows became pregnant. The test was repeated without these 2 groups of cows to check whether their inclusion influenced the difference between the mean prognosis and mean insemination success.

\section{RESULTS}

Table 2 shows the descriptive statistics of the variables in the data set. On average, cows were inseminated for the first time at 96 DIM of a lactation and the average insemination success was $42.8 \%$. The average milk yield at the time of insemination was $32.9 \mathrm{~kg} / \mathrm{d}$.

All of the independent variables decreased the AIC of the prognostic model in the forward selection conducted in statistical analysis 1 (Table 3). Therefore, all variables were selected and the model contained the following variables: random intercept for farm effect, parity, DIM at insemination, log of the ratio of fat to protein, days to peak milk yield, relative production factor, BCS at insemination, milk yield at insemination, breed of the cow, insemination season, and calving season. No variables were excluded in the backward exclusion procedure. The variables that decreased the AIC most were the random farm effect $(1,653)$, relative production factor (867), and milk yield at insemination (724). The smallest AIC decrease was observed for insemination season (9), followed by DIM (33) and calving season (53). To give a prognosis of the success of insemination, farm effects are most important and insemination season is the least important.

The parameter estimates for the parametric regression analysis for the prognostic model (statistical analysis 1) and the bootstrapped regression (statistical analysis 2) are shown in Table 4. For the bootstrapped regression, the parameter estimates for all bootstrap iterations are summarized by the mean, median, and standard error. The concept of a bootstrap procedure is that inference of a statistic can be approximated by resampling the true population. The highest bias and largest confidence intervals were observed for the intercept and BCS at insemination (Table 4). In general, the bias among the parameter estimates ranged between 0.000 and 0.009 . The bias is the approximated difference between the expected value and its population value, and describes how strongly an estimate might be under- or over-estimated.

Confidence intervals were calculated for the mean value of the parameters estimates from the bootstrap iterations. These bootstrapped confidence intervals were small for most parameter estimates; for instance, the bootstrapped $95 \%$ CI was 0.053 to 0.059 for DIM. The low biases and small confidence intervals indicate appropriately estimated coefficients; therefore, the prognostic model can generally be expected to provide appropriate prognoses in practice.

Figure 1 shows bootstrapped variation in the coefficients for BCS at insemination and $\log$ of the ratio of fat to protein. The boxplot for the parameter estimates for the ratio of fat to protein was rather small, indicating that estimates changed only slightly across different bootstrap samples. The boxplot for the parameter estimates for BCS at insemination was much larger. Some outliers were also observed outside the boxplots for BCS. This indicates that sampling a different group of farms from the data set causes variation 
Table 3. Comparison of Akaike information criterion (AIC) values for the forward selection of variables for the mixed logistic model (statistical analysis 1 ) and the contribution of each variable to model fit (comparison of AIC values in a backward elimination)

\begin{tabular}{|c|c|c|c|}
\hline \multirow[b]{2}{*}{$\begin{array}{l}\text { Variable added } \\
\text { to the model }\end{array}$} & \multirow[b]{2}{*}{$\mathrm{AIC}$} & \multicolumn{2}{|c|}{ Change in AIC: } \\
\hline & & $\begin{array}{l}\text { After adding } \\
\text { a variable }\end{array}$ & $\begin{array}{l}\text { After removing a } \\
\text { variable from the } \\
\text { end } \text { model }^{2}\end{array}$ \\
\hline $\mathrm{Null}^{3}$ & 260,163 & $\mathrm{NA}^{4}$ & NA \\
\hline Random ${ }^{5}$ & 258,012 & $-2,151$ & 1,653 \\
\hline Parity & 256,802 & $-1,210$ & 146 \\
\hline DIM & 256,750 & -52 & 33 \\
\hline Log of ratio fat to protein & 256,715 & -36 & 109 \\
\hline Days to peak milk yield & 256,638 & -77 & 138 \\
\hline Relative production factor ${ }^{6}$ & 256,404 & -234 & 867 \\
\hline BCS & 256,278 & -126 & 89 \\
\hline Milk yield & 255,576 & -702 & 724 \\
\hline Cow breed & 255,453 & -123 & 116 \\
\hline Insemination season & 255,372 & -81 & 9 \\
\hline Calving season & 255,319 & -53 & 53 \\
\hline \multicolumn{4}{|c|}{$\begin{array}{l}\text { 19 the forward selection, variables were added to the mixed logistic model in descending order (starting with } \\
\text { the "null" model and ending with adding "calving season"). In each step, the AIC after adding a variable was } \\
\text { compared with the AIC of the previous step. }\end{array}$} \\
\hline \multicolumn{4}{|c|}{$\begin{array}{l}{ }^{2} \text { The end model is the model resulting from the forward selection. In the backward elimination, each variable } \\
\text { was removed from the end model and the effect on the AIC was estimated. }\end{array}$} \\
\hline \multicolumn{4}{|c|}{$\begin{array}{l}{ }^{3} \text { Logistic model without random effect and with an intercept as the only independent variable. } \\
{ }^{4} \text { Not applicable. }\end{array}$} \\
\hline${ }^{5}$ Mixed logistic model with & 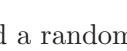 & pt for farm effe & \\
\hline
\end{tabular}

in the parameter estimates. Furthermore, the boxplots of the parameter estimates for BCS levels from 1.5 to 4.5 show an ascending line. This indicates that a higher BCS at insemination has a positive effect on the likelihood of insemination success. In contrast, the parameter estimate for a BCS of 5 at insemination is lower than the parameter estimates for a BCS of 3.5 to 4.5 , indicating a negative effect on the likelihood of insemination success. The boxplot for the parameter estimate was also much larger for a BCS of 5 than for the other levels. The mean estimated coefficients for BCS might therefore not be appropriate for prognostic purposes in some cows.

Three of the additional variables in statistical analysis $3, \log$ of SCC, lactose percentage, and ketosis, decreased the AIC value (Table 5). None of these variables were removed in the backward elimination. In contrast, addition of log of urea content increased the model's AIC, and this variable was therefore not selected for the model. All AIC values in Table 5 were lower than the values from the forward selection in statistical analysis 1 (Table 3) because the models used for Table 5 were fitted on a smaller data set.

The AIC for the full model with the ratio of fat to protein calculated from values of the previous milk recording was 255,319 . The AIC for the full model with the ratio calculated from interpolated fat and protein contents was lower, at 254,757, a difference of 562 .

The parameter estimates for the parametric regression analysis for the prognostic model (statistical analysis 3 ) and the bootstrapped regression (statistical analysis 4) for the second data set are shown in Table 6 . The biases for the variables $\log$ of SCC, lactose content, and ketosis score were $-0.194,-0.012$, and 0.195 , respectively. The mean bootstrapped parameter estimates for BCS at insemination ranged from -0.79 to 1.203.

In Table 7, mean insemination success and mean prognosis of insemination success are presented for each class level of parity, BCS at insemination, hair color, insemination season, and calving season. For each parity group, the mean prognosis was slightly lower than the mean insemination success and all median prognoses were slightly lower than the mean prognoses. For instance, for the parity group $5+$, the median prognosis was $35.15 \%$, whereas the mean prognosis was $35.59 \%$. For each level of BCS at insemination, there was a slight difference between the mean prognosis and the mean insemination success. The mean insemination success and mean prognosis were higher for a BCS of 4.5 than for other levels of BCS. The difference between mean prognosis $(46.88 \%)$ and mean insemination success $(46.18 \%)$ was -0.70 percentage points for red and 
white cows. This difference was slightly larger for cows with other hair color, where the difference between mean prognosis $(52.06 \%)$ and mean insemination success $(50.82 \%)$ was -1.24 percentage points. For the 5 parity groups, the probability density of each prognosis on insemination success is described graphically in Figure 2. In each parity group, about half of the cows had a prognosis for insemination success above the average insemination success. The probability density function for parity 1 was slightly smaller than that of other parities.
The value of the test statistic, V, was 531,435 for the Wilcoxon signed rank test for statistical analysis 1 , with a corresponding $P$-value of 0.258 . For statistical analysis 2 , the value of the test statistic $\mathrm{V}$ was 367,813 with a corresponding $P$-value of 0.251 . Therefore, no significant difference was observed at the $5 \%$ level.

Table 8 shows the median value of some variables in the data set for each level of BCS at insemination. Cows with a BCS between 2 and 3.5 formed the largest groups of cows. Cows inseminated at a low BCS (1-2) were, in general, older than the other cows (parity 3

Table 4. Parameter estimates for the parametric and bootstrapped mixed logistic regressions, with the estimates of bias and variation from the bootstrapped regression (statistical analysis 2)

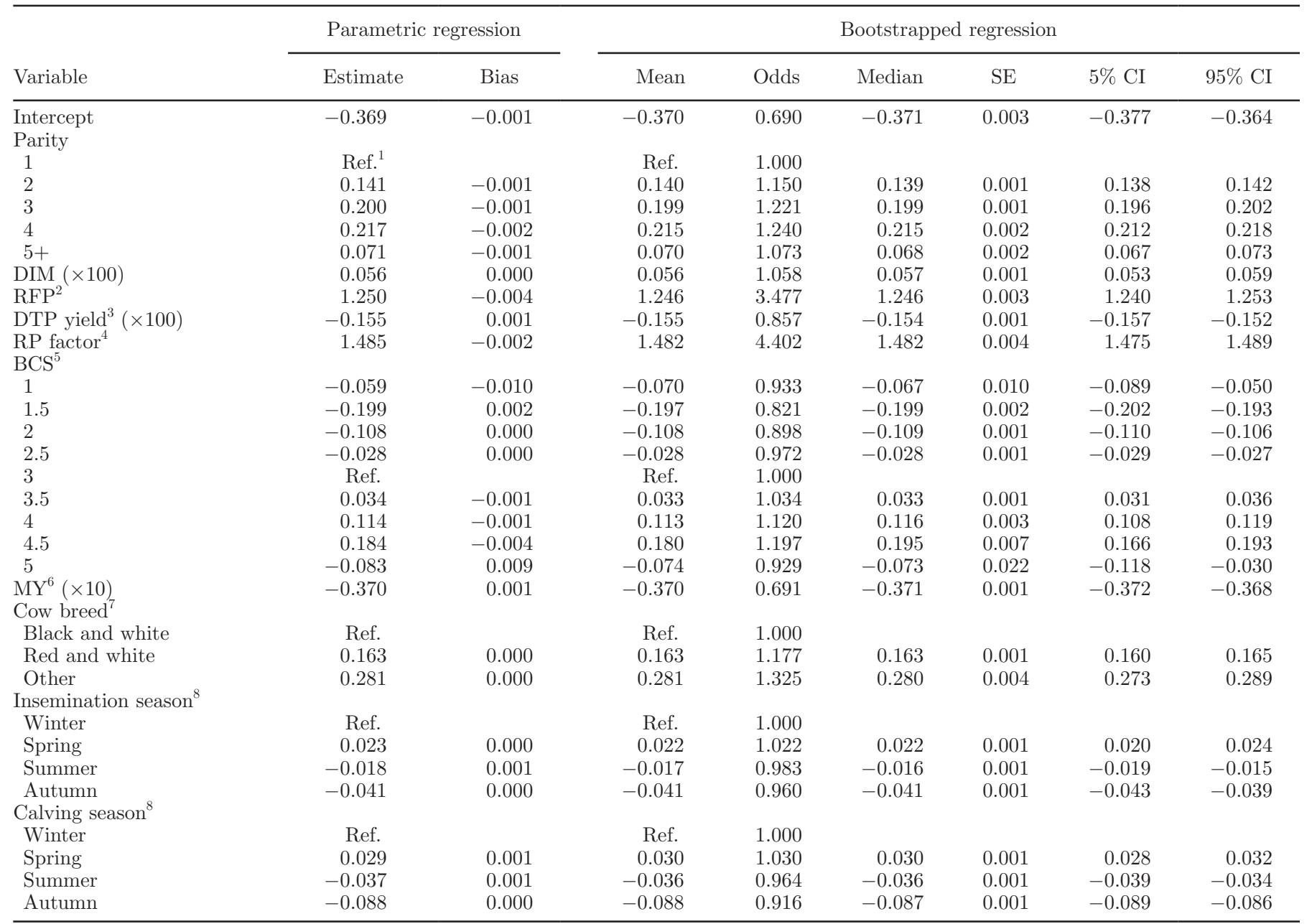

\footnotetext{
${ }^{1}$ Reference level for the variable.

${ }^{2} \log$ of the ratio of fat to protein content at the last milk recording before insemination.

${ }^{3}$ Days to peak milk yield.

${ }^{4}$ Relative production factor, a factor that represents the production level of an individual cow relative to her herd mates.

${ }^{5} \mathrm{BCS}$ scored on a 5 -point scale at the time of insemination.

${ }^{6}$ Daily milk yield on the day of insemination.

${ }^{7}$ Breed is defined as black and white (Holstein), red and white (Holstein or Dutch red and white), or other. Black and white is the reference level for this variable.

${ }^{8}$ Season is defined as spring (March-May), summer (June-August), autumn (September-November), or winter (December-February). Winter is the reference level for this variable.
} 
Table 5. Continued forward selection and backward elimination (statistical analysis 3) on a smaller data set for the variables log of SCC, lactose content (\%), log of urea, and ketosis score; model comparison was based on Akaike information criterion (AIC)

\begin{tabular}{lccc}
\hline & & \multicolumn{2}{c}{ Change in AIC: } \\
\cline { 3 - 4 } Variable & AIC & $\begin{array}{c}\text { After adding } \\
\text { a variable }\end{array}$ & $\begin{array}{c}\text { After removing a } \\
\text { variable from the } \\
\text { end model }^{2}\end{array}$ \\
\hline $\begin{array}{l}\text { End model of statistical analysis 1 } \\
\text { Log of SCC }\end{array}$ & 100,853 & $\mathrm{NA}^{3}$ & $\mathrm{NA}$ \\
Lactose content (\%) & 100,828 & -26 & 18 \\
Ketosis score & 100,823 & -5 & 3 \\
Log of urea & 100,811 & -12 & 12 \\
\hline
\end{tabular}

${ }^{1}$ In the forward selection, variables were added to the mixed logistic model in descending order (starting with the "end" model of statistical analysis 1 and ending with adding "ketosis score"). In each step, the AIC after adding a variable was compared with the AIC of the previous step.

${ }^{2}$ The end model is the model resulting from the forward selection. In the backward elimination, each variable was removed from the end model and the effect on the AIC was estimated.

${ }^{3}$ Not applicable.

${ }^{4}$ This variable was not selected for the prognostic model because the AIC value increased after addition of the variable in the forward selection.

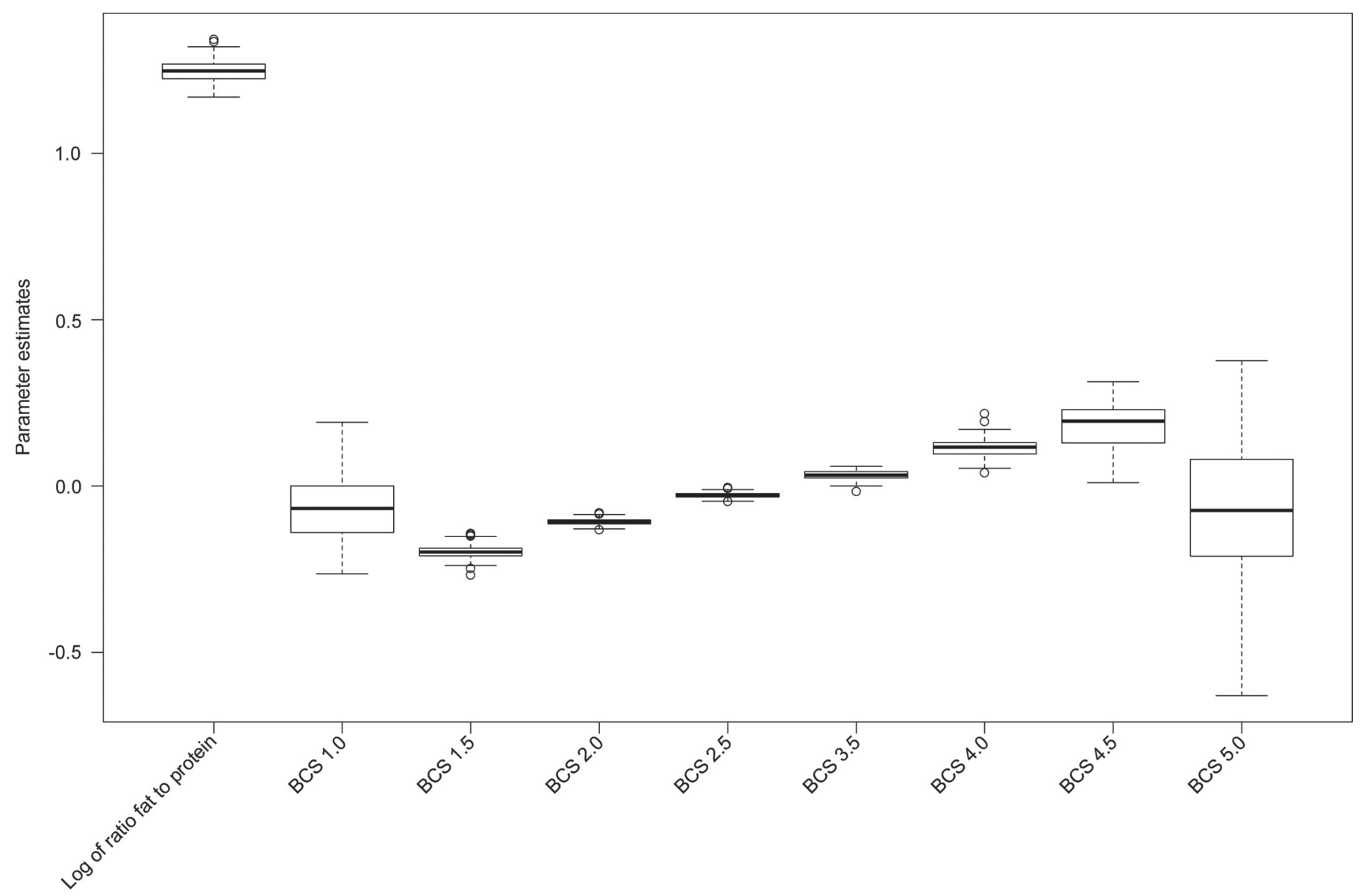

Figure 1. Boxplots of the distributions of the bootstrapped coefficients for 2 variables in the prognostic model for the likelihood of a successful first insemination: $\log$ of the ratio of fat to protein and BCS at insemination. The lines represent the 25th, 50th (median), and 75th percentiles; the whiskers extend to 1.5 times the interquartile distance. Values outside the whiskers were considered outliers and are represented by dots. 
Table 6. Parameter estimates of the parametric and bootstrapped mixed logistic regressions for the second data set, including SCC, lactose content, and ketosis score, with the estimates of bias and variation from the bootstrapped regression (statistical analysis 4)

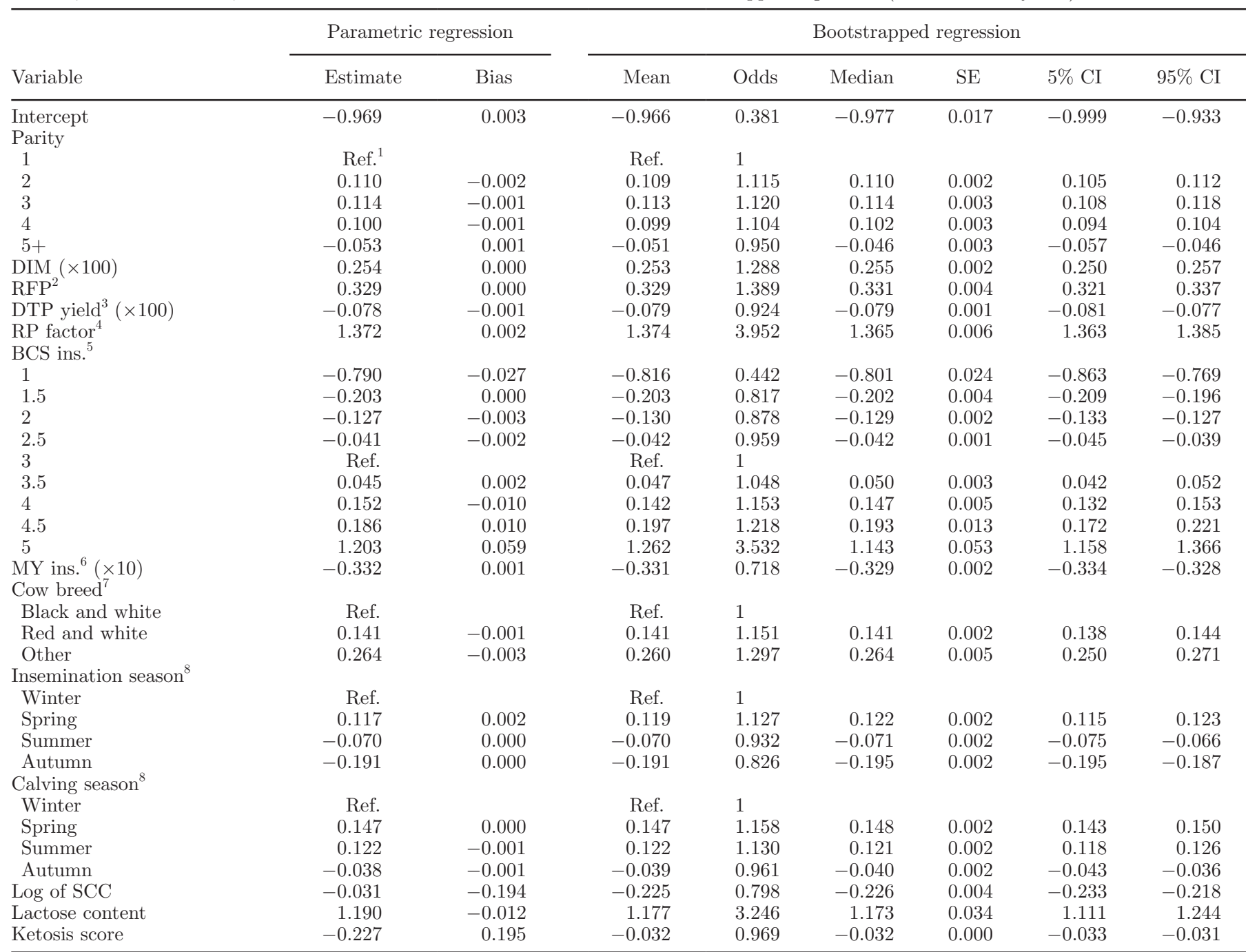

${ }^{1}$ Reference level for this variable.

${ }^{2} \log$ of the ratio of fat to protein content at the last milk recording before insemination.

${ }^{3}$ Days to peak milk yield.

${ }^{4}$ Relative production factor, a factor that represents the production level of an individual cow relative to her herd mates.

${ }^{5}$ BCS scored on a 5-point scale at the time of insemination.

${ }^{6}$ Daily milk yield at the day of insemination.

${ }^{7}$ Breed is defined as black and white (Holstein), red and white (Holstein or Dutch red and white), or other. Black and white is the reference level for this factor.

${ }^{8}$ Season is defined as spring (March-May), summer (June-August), autumn (September-November), or winter (December-February). Winter is the reference level for this factor.

vs. parity 2). In general, cows with a lower BCS had a higher DIM at insemination, with the exception of a BCS of 5 .

\section{DISCUSSION}

The overall percentage of successful first inseminations was $42.8 \%$, which is in line with previous studies in the Netherlands (Windig et al., 2005; Inchaisri et al., 2010a). The first insemination was, on average, observed at 96 DIM. Under Dutch conditions, a VWP of 84 DIM was assumed realistic (Inchaisri et al., 2010b), and a first insemination at 96 DIM seems to fit with this VWP. The variables contributing most to prognosis were farm effect, relative production factor, and milk yield at the time of insemination. The odds ratios 
Table 7. Observed insemination success (mean value) and the prognosis of insemination success (mean, median, 10th and 90th percentiles), for each group of cows grouped according to the class variables

\begin{tabular}{|c|c|c|c|c|c|c|}
\hline Group & $\frac{\text { AI success }(\%)}{\text { Mean }}$ & \multicolumn{4}{|c|}{ Prognosis (\%) } & No./iteration \\
\hline 1 & 47.41 & 47.26 & 47.04 & 41.40 & 53.46 & 19,760 \\
\hline 2 & 43.58 & 43.33 & 43.12 & 36.66 & 50.25 & 16,725 \\
\hline 3 & 41.03 & 40.74 & 40.48 & 33.93 & 47.82 & 11,176 \\
\hline 4 & 39.61 & 39.28 & 38.98 & 32.43 & 46.51 & 7,181 \\
\hline 1.0 & 38.44 & 39.02 & 38.92 & 30.39 & 48.03 & 56 \\
\hline 1.5 & 36.81 & 36.80 & 36.43 & 29.20 & 44.92 & 1,459 \\
\hline 2.0 & 40.12 & 40.05 & 40.05 & 31.89 & 48.12 & 12,110 \\
\hline 2.5 & 43.04 & 42.78 & 42.92 & 34.53 & 50.63 & 29,377 \\
\hline 3.0 & 44.22 & 43.90 & 44.16 & 35.40 & 51.86 & 16,207 \\
\hline 3.5 & 45.63 & 45.21 & 45.38 & 36.30 & 53.64 & 3,365 \\
\hline 4.0 & 48.09 & 47.57 & 47.80 & 38.77 & 56.01 & 665 \\
\hline Other & 52.06 & 50.82 & 51.30 & 42.43 & 58.52 & 695 \\
\hline \multicolumn{7}{|c|}{ Insemination season } \\
\hline Winter & 42.06 & 41.73 & 41.85 & 32.98 & 50.11 & 16,228 \\
\hline Spring & 43.33 & 43.03 & 43.26 & 34.33 & 51.22 & 16,585 \\
\hline Summer & 43.68 & 43.53 & 43.72 & 34.93 & 51.69 & 13,935 \\
\hline Autumn & 42.42 & 42.23 & 42.31 & 33.70 & 50.45 & 16,644 \\
\hline \multicolumn{7}{|l|}{ Calving season } \\
\hline Winter & 43.61 & 43.27 & 43.49 & 34.54 & 51.44 & 17,333 \\
\hline Spring & 44.01 & 43.87 & 44.11 & 35.37 & 51.87 & 13,740 \\
\hline Summer & 42.31 & 42.18 & 42.21 & 33.70 & 50.49 & 14,929 \\
\hline Autumn & 41.61 & 41.28 & 41.43 & 32.69 & 49.50 & 17,390 \\
\hline
\end{tabular}

${ }^{1}$ The average group size because group sizes could differ from each bootstrap iteration to another.

for breed were comparable to those found by Inchaisri et al. (2010a). The other effects are in line with previous research. For instance, a higher parity, smaller number of DIM, and higher milk yield decrease the likelihood of a successful insemination (Roche et al., 2009; Norman et al., 2010; Inchaisri et al., 2011a; Bello et al., 2012; Hernandez et al., 2012). Some deviations in the results compared with other studies can be attributed to differences between the studies, such as the consideration of repeated inseminations (Inchaisri et al., 2011a) or only non-Holstein cows (Schneider et al., 2007). Furthermore, the current study focused on the development of a prognostic model, not on a detailed analysis of individual risk factors.

For the current analysis, a large data set was obtained. However, some data were not useful as information was missing, such as calving dates that marked the start of the lactation in which a cow was inseminated. This was mainly caused by the selection of the data from a specific period. It is unlikely that this caused a selection bias, as data missed for all cows in the same period. The fitting of lactation curves resulted in an important data reduction because either too few milk recordings were available or a nonaberrant curve was fitted. Both reasons for exclusion might be related to health problems. Cows that were culled early in lactation would not have the required number of milk recordings available. Health problems would be a likely reason, because cows that, for instance, failed to conceive would most likely finish their lactation before culling. Health problems (e.g., a caesarean section or a severe case of clinical mastitis) cause strong and sudden drops in milk production that disrupt the course expected for a normal lactation curve. Registration of diseases was not available so correction for such shocks and incorporation in the prognostic model was not possible. The reason is that recording diseases is optional for farmers; therefore, disease records are not as complete and reliable as milk recording and insemination data. These 2 selections of the data cause the model to be unsuitable for cows that experience a severe disease episode. Finally, an important data reduction was the focus on the first insemination within a lactation. This selection of data did fit the aim of the current study best. 
Table 8. Median values of variables milk yield, parity, and DIM at first insemination with the respective number of records (n) for each level of BCS at insemination

\begin{tabular}{|c|c|c|c|c|}
\hline BCS & $\mathrm{n}$ & $\begin{array}{l}\text { Milk yield } \\
(\mathrm{kg} / \mathrm{d})\end{array}$ & Parity & DIM \\
\hline 1.0 & 168 & 33.45 & 3 & 92 \\
\hline 1.5 & 4,366 & 34.33 & 3 & 93 \\
\hline 2.0 & 36,372 & 33.6 & 3 & 89 \\
\hline 2.5 & 88,313 & 32.69 & 2 & 86 \\
\hline 3.0 & 48,713 & 32.03 & 2 & 83 \\
\hline 3.5 & 10,121 & 30.68 & 2 & 82 \\
\hline 4.0 & 2,004 & 29.78 & 2 & 81 \\
\hline 4.5 & 411 & 29.42 & 2 & 80 \\
\hline 5.0 & 45 & 27.63 & 2 & 99 \\
\hline
\end{tabular}

Etiological models assess causality of individual factors for a condition (e.g., a disease), whereas diagnostic models determine the condition based on symptoms. Prognostic modeling differs from etiologic and diagnostic models in the sense that the sole focus is to give a prognosis on future development (Grobbee and Hoes, 2015). Prognostic models are therefore mainly evaluat- ed by the accuracy of the prognosis (Grobbee and Hoes, 2015). Furthermore, effects such as confounding are ignored, because they have no relevance for the accuracy of a prognosis. For the current study, recommendations on reporting a prognostic study were considered in a TRIPOD statement, which was originally designed for predictive modeling in human medicine (Moons et al., 2015; Data Supplement; http://dx.doi.org/10.3168/ jds.2016-10935). Prognostic models are frequently used in clinical epidemiology, mainly in human medicine; for example, the prognosis of therapy success for colon cancer (Hugen et al., 2015). The purpose of prognostic modeling is to generate a prognosis (or expectation) of further or future development of a disease; for instance, a prognosis of the success of surgery on a dislocated abomasum in dairy cattle (Croushore et al., 2013; Buczinski et al., 2015). A prognostic approach was considered appropriate in this study because practical decision-making will be influenced by the expected outcome and reliability of the expectation, not by the causal relations that may play a role.
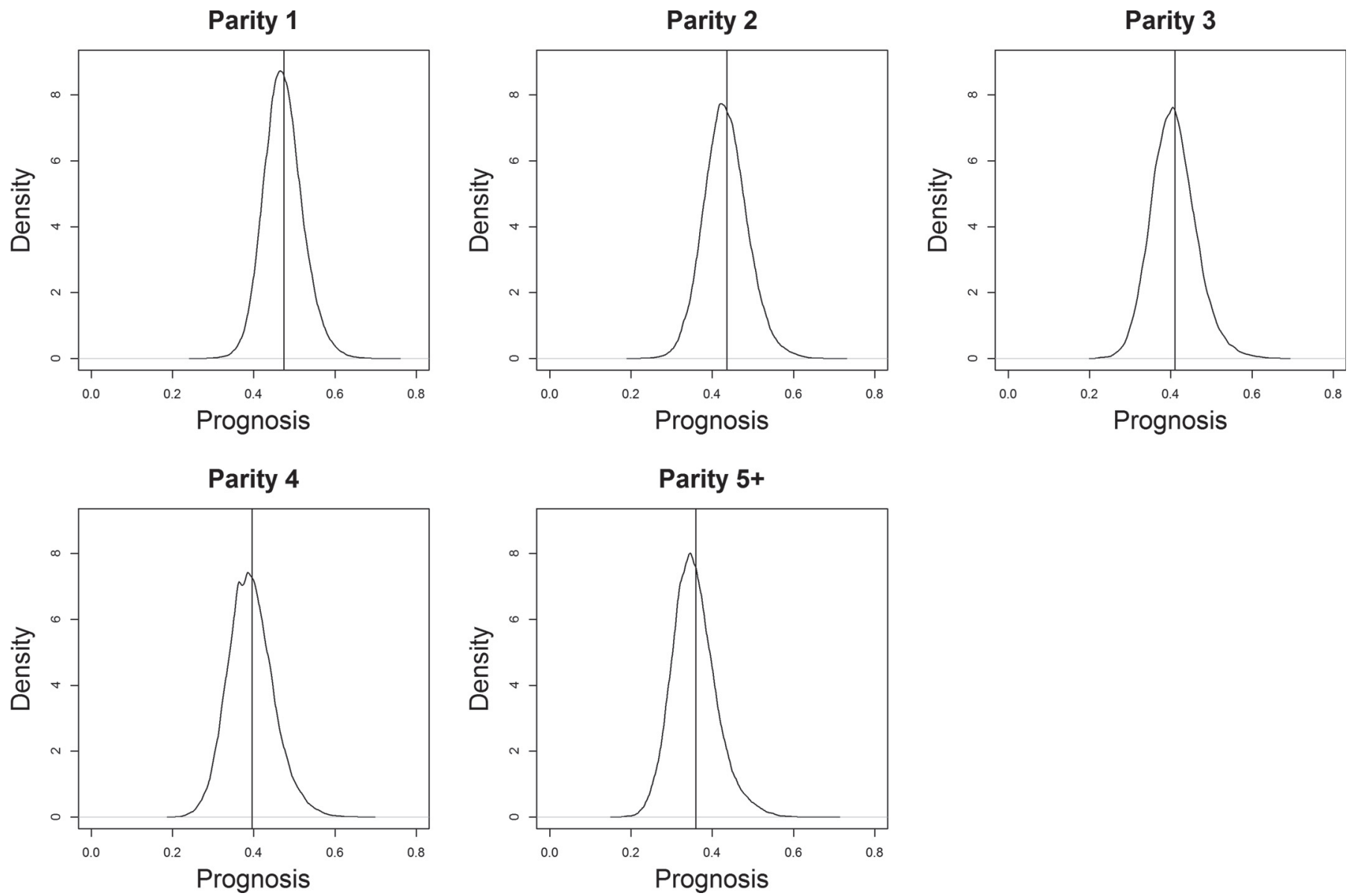

Figure 2. Probability density functions of the prognosis for insemination success, with average insemination success (solid vertical line) for each parity group. 
Previous studies have demonstrated the application of machine learning algorithms on the prediction of insemination success (Caraviello et al., 2006; Shahinfar et al., 2014). Machine learning algorithms are powerful solutions to classification problems (Shahinfar et al., 2014). In general, machine learning algorithms use an adaptive approach of model selection and cross validation to find the model that most correctly assigns individuals to a class (Hastie et al., 2009). For the prognostic approach, explicit classification was not the goal but rather the estimation of a continuous probability. Furthermore, logistic regression enables the individual evaluation of variables in terms of model contribution and odds ratios. However, logistic regression requires a separate independent validation, which was conducted in the bootstrap analysis that also assessed potential bias.

In general, prognostic studies give a prognosis of a disease outcome, such as life expectancy or pain level, on a continuous scale that is divided into classes. The accuracy of the model is then evaluated by analyzing how accurate the prognosis assigns patients to their respective classes. More diagnostic models focus on a binary outcome, such as whether surgery for displaced abomasum for an individual cow was successful. These models give a likelihood, which is then classified on a binary scale. Both methods were considered inappropriate for the current model. Our focus was on estimation of a prognosis of insemination success, rather than predicting insemination success for individual cows. This means the model estimates a likelihood of success, which could not be validated on an individual cow level because the insemination outcome (pregnant or not) was a binary outcome and not a probability. Therefore, the validation was conducted for groups of cows with similar characteristics. This means that groups were defined only based on categorical variables, but all variables of specific cows within these groups were used to estimate the prognosis.

Mean prognosis resembled mean insemination success, as no statistical difference between the 2 variables could be observed. The Wilcoxon signed rank test was repeated for groups of at least 100 cows and for a set of groups, where all groups containing either only pregnant or only non-pregnant cows were excluded. The aim of this repetition was to see if these 2 groups might have influenced the results. No significant difference between the mean prognosis of insemination success and mean insemination success was found for these additional tests.

The difference in AIC between the 2 models with different methods for calculating the ratio of fat to protein (interpolated values versus the values of the last milk recording) was larger than the differences observed in the forward selection and backward elimination. This indicates that the time between insemination and the date at which fat and protein contents are measured is important for a prognostic model of the likelihood of insemination success. Interpolated fat and protein contents cannot be used in practice, as the curve for these factors over a lactation is not available. An alternative to the last milk recording, which more closely approximates the fat and protein contents on the day of insemination, could improve the prognosis of the model. Options might be fat and protein content estimated by inline sensors in the milking equipment or a predicted value based on milk recordings and milk yield (Brandt et al., 2010). The reliability in practice of such alternatives to milk recording data will be important to achieve an improvement in the prognosis of insemination success.

In general, the mean and median prognosis of insemination success were some tenths of a percentage lower than the mean insemination success. This indicates that the prognostic model tended to slightly underestimate the likelihood of insemination success. However, the differences were considered small, which indicates no problem in applying the model in practice. The underestimations were greater than $0.5 \%$ for the classes BCS 4 and 4.5, red and white cows, and cows of a different hair color. Cows with a BCS of 1, 1.5, and especially 5 were, in general, inseminated later than other cows. Cows with a BCS of 1 had a lower milk production than cows with a BCS of 1.5, which could explain the lower prognosis of insemination success of the latter group. The prognosis on insemination success was still $>35 \%$ for BCS 1 and 1.5, although a low BCS is often associated with low insemination success (Roche et al., 2009). Three things are important to note here. First, the number of records was small for BCS 1 and 1.5. Second, the change in BCS, which is biologically related to fertility, was not assessed and BCS might be less accurate for thin cows (Roche et al., 2009). Third, farmers might have specific reasons for inseminating these cows regardless of their BCS; for instance, because they received fertility treatment. The difference between the median and mean prognosis was less than 0.3 percentage points for most classes, indicating that the skewedness of the distributions of prognosis was limited and the respective confidence intervals were small. The difference between mean and median prognosis was relatively high for red and white cows (0.3 percentage points) and for cows with another hair color (0.48 percentage points). This might indicate that cows in these 2 classes of hair color have a strong influence on the average prognosis. All these findings suggest 
that the prognostic model produces a useful prognosis of the likelihood of insemination success. Some caution seems appropriate, however, for cows that are not black and white, and cows with an extreme BCS $(1,1.5,4$, 4.5 , or 5$)$ at insemination. For these cows, prognosis might be over- or underestimated in practice.

Changes in a cow's BCS are important approximations of a cow's energy balance (Roche et al., 2009). The cow's energy balance influences her fertility and BCS is related, therefore, to insemination success (Roche et al., 2009; Bello et al., 2012; Hernandez et al., 2012). Only BCS at insemination was available in the current data set, which is an estimate at a single moment in time. Body condition score at insemination did contribute to the prognostic model and its parameter estimates were robust, but this contribution was small compared with other variables. Moreover, BCS at insemination might not be an accurate approximation of the cow's energy balance, as the change in BCS more accurately reflects energy balance than the BCS at a single point in lactation (Roche et al., 2009). In contrast to BCS, the $\log$ of the ratio of fat to protein decreased the model's AIC more and thus gave a more direct indication of the cow's energy balance. For a prognostic model, this might mean that a better prognosis could be possible if the cow's energy balance could be approximated more accurately. Nevertheless, BCS and milk production provide proxies of underlying diseases that affect fertility in dairy cows. Diseases are known to influence insemination success (Inchaisri et al., 2010a; Shahinfar et al., 2015).

For farm management, the likelihood of insemination success is important for the economic outcome of breeding decisions (Tenhagen et al., 2004; De Vries, 2006; Olynk and Wolf, 2009). For instance, when the prognosis of insemination success is low (e.g., 0.25), a farmer might want to postpone the start of breeding (depending on the lactation stage) or use a (beef) bull with a higher conception rate. Alternatively, a farmer might want to use sexed semen on cows with a high prognosis of insemination success. Farmers could compare the prognosis on insemination success to the average AI success observed in a group of similar cows; for instance, in the same parity, as illustrated by the graphs of Figure 2. These decisions could be economically optimized in a decision support model, such as the model developed by Steeneveld and Hogeveen (2012). Previous work has indicated that the VWP (Inchaisri et al., 2011b), the decision to stop inseminating cows (Inchaisri et al., 2012), and inseminating only a proportion of the herd with the highest chance on insemination success could be optimized economically (Shahinfar et al., 2015). Therefore, the potential exists for economi- cally optimized decision support systems for insemination decisions, which would need a prognostic model like the current model.

In addition, sensor technology has developed rapidly in recent years, and one of the most applied technologies is automated estrus detection, although integrated decision support systems are lacking (Rutten et al., 2013). Activity meters can improve estrus detection over that achieved by the farmer (Kamphuis et al., 2012; Rutten et al., 2014). Better estrus detection enables farmers to register estrus automatically and thereby determine more easily whether cows exhibit a normal estrus cycle. Furthermore, a better detection rate increases the chance that a subsequent estrus will be detected and therefore postponing an insemination will have a lower risk of missing the next estrus. The downside of using automated estrus detection is that such a system will produce false positive alerts, which may cause famers to inseminate cows that are not actually in estrus. A prognostic model could be integrated in a decision support system that uses information of an automated estrus detection system. The information of the prognostic model could be used to produce additional information that could help farmers identify false positive alerts of the automated estrus detection system. One type of automated estrus detection is of special interest; that is, a sensor that measures progesterone levels in milk (Østergaard et al., 2005; Käppel et al., 2007). For a prognostic model, these progesterone measurements might not only be useful for estrus detection but also for prognosis of insemination success. A stronger decrease in progesterone level might indicate a better prognosis for insemination success.

\section{CONCLUSIONS}

Routinely collected farm data and test-day milk yield records have value for the prognosis of insemination success in dairy cows. The prognosis of insemination success closely resembled the mean insemination success observed in the data set. The most important variables in this prognostic model were farm effect, relative production factor, and milk yield at insemination. The variables SCC, urea, and lactose percentage also contributed to the prognostic power of the model. Robust estimates can be obtained for most parameters in the prognostic model.

\section{ACKNOWLEDGMENTS}

This research was supported by the Dutch research program Smart Dairy Farming, which is financed by Royal Friesland Campina (Amersfoort, the Nether- 
lands), CRV (Arnhem, the Netherlands), Royal Agrifirm (Apeldoorn, the Netherlands), Dairy Valley (Leeuwarden, the Netherlands), Investment and Development Agency for the Northern Netherlands (Groningen, the Netherlands), the Dutch Dairy Board (Zoetermeer, the Netherlands), and the ministry of Economic Affairs, Agriculture and Innovation, Pieken in de Delta (Den Haag, the Netherlands).

\section{REFERENCES}

Bates, D., M. Maechler, B. Bolker, and S. Walker. 2015. Fitting linear mixed-effects models using \{lme4\}. J. Stat. Softw. 67:1-48. 10.18637/jss.v067.i01.

Bello, N. M., J. S. Stevenson, and R. J. Tempelman. 2012. Invited review: Milk production and reproductive performance: Modern interdisciplinary insights into an enduring axiom. J. Dairy Sci. 95:5461-5475.

Brandt, M., A. Haeussermann, and E. Hartung. 2010. Invited review: Technical solutions for analysis of milk constituents and abnormal milk. J. Dairy Sci. 93:427-436.

Buczinski, S., G. Boulay, and D. Francoz. 2015. Preoperative and postoperative L-lactatemia assessment for the prognosis of right abomasal disorders in dairy cattle. J. Vet. Intern. Med. 29:375-380.

Canu, S., M. Boland, G. M. Lloyd, M. Newman, M. F. Christie, P. J. May, R. M. Christley, R. F. Smith, and H. Dobson. 2010. Predisposition to repeat breeding in UK cattle and success of artificial insemination alone or in combination with embryo transfer. Vet. Rec. 167:44-51.

Caraviello, D. Z., K. A. Weigel, M. Craven, D. Gianola, N. B. Cook, K. V. Nordlund, P. M. Fricke, and M. C. Wiltbank. 2006. Analysis of reproductive performance of lactating cows on large dairy farms using machine learning algorithms. J. Dairy Sci. 89:4703-4722.

Croushore, W. S., P. A. Ospina, D. C. Welch, D. J. Zawisza, and D V. Nydam. 2013. Association between beta-hydroxybutyrate concentration at surgery for correction of left-displaced abomasum in dairy cows and removal from the herd after surgery. J. Am. Vet. Med. Assoc. 243:1329-1333.

de Roos, A. P. W., H. van den Bijgaart, J. Horlyk, and G. de Jong. 2007. Screening for subclinical ketosis in dairy cattle by Fourier transform infrared spectrometry. J. Dairy Sci. 90:1761-1766.

De Vries, A. 2006. Economic value of pregnancy in dairy cattle. J. Dairy Sci. 89:3876-3885.

Dematawewa, C. M. B., R. E. Pearson, and P. M. VanRaden. 2007. Modeling extended lactations of Holsteins. J. Dairy Sci. 90:39243936

Dijkstra, J., J. France, M. S. Dhanoa, J. A. Maas, M. D. Hanigan, A J. Rook, and D. E. Beever. 1997. A model to describe growth patterns of the mammary gland during pregnancy and lactation. J. Dairy Sci. 80:2340-2354.

Dowle, M., T. Short, and S. Lianoglou. 2013. Data.table: Extension of data.frame. Accessed May 18, 2016. https://github.com/ Rdatatable/data.table/wiki.

Elzhov, T. V., K. M. Mullen, A. N. Spiess, and B. Bolker. 2013. minpack.lm: R Interface to the Levenberg-Marquardt nonlinear leastsquares algorithm found in MINPACK, plus support for bounds. Accessed May 18, 2016. https://cran.r-project.org/web/packages/ minpack.lm/index.html.

Fourichon, C., H. Seegers, and X. Malher. 2000. Effect of disease on reproduction in the dairy cow: A meta-analysis. Theriogenology 53:1729-1759.

Gábor, G., F. Toth, L. Ozsvari, Z. Abonyi-Toth, and R. G. Sasser. 2008. Factors influencing pregnancy rate and late embryonic loss in dairy cattle. Reprod. Domest. Anim. 43:53-58.

Giordano, J. O., P. M. Fricke, M. C. Wiltbank, and V. E. Cabrera 2011. An economic decision-making support system for selection of reproductive management programs on dairy farms. J. Dairy Sci. 94:6216-6232.
Grobbee, D. E., and A. W. Hoes. 2015. Clinical Epidemiology: Principles, Methods and Applications for Clinical Research. 2nd ed. Jones and Bartlett Learning, Burlington, MA.

Hastie, T., R. Tibshirani, and J. Friedman. 2009. The Elements of Statistical Learning: Data Mining, Inference and Prediction. 2nd ed. Springer Series in Statistics. Springer-Verlag, New York, NY.

Hernandez, J. A., C. A. Risco, F. S. Lima, and J. E. P. Santos. 2012 Observed and expected combined effects of clinical mastitis and low body condition on pregnancy loss in dairy cows. Theriogenology $77: 115-121$

Hugen, N., R. H. Verhoeven, V. E. Lemmens, C. J. van Aart, M. A. Elferink, S. A. Radema, I. D. Nagtegaal, and J. H. de Wilt. 2015. Colorectal signet-ring cell carcinoma: Benefit from adjuvant chemotherapy but a poor prognostic factor. Int. J. Cancer 136:333-339.

Inchaisri, C., A. De Vries, R. Jorritsma, and H. Hogeveen. 2012 Improved knowledge about conception rates influences the decision to stop insemination in dairy cows. Reprod. Domest. Anim. $47: 820-826$.

Inchaisri, C., H. Hogeveen, P. Vos, G. C. van der Weijden, and R. Jorritsma. 2010a. Effect of milk yield characteristics, breed, and parity on success of the first insemination in Dutch dairy cows. J. Dairy Sci. 93:5179-5187.

Inchaisri, C., R. Jorritsma, J. C. M. Vernooij, P. Vos, G. C. van der Weijden, and H. Hogeveen. 2011a. Cow effects and estimation of success of first and following inseminations in Dutch dairy cows. Reprod. Domest. Anim. 46:1043-1049.

Inchaisri, C., R. Jorritsma, P. Vos, G. C. van der Weijden, and H. Hogeveen. 2010b. Economic consequences of reproductive performance in dairy cattle. Theriogenology 74:835-846.

Inchaisri, C., R. Jorritsma, P. Vos, G. C. van der Weijden, and H. Hogeveen. 2011b. Analysis of the economically optimal voluntary waiting period for first insemination. J. Dairy Sci. 94:3811-3823.

Kamphuis, C., B. DelaRue, C. R. Burke, and J. Jago. 2012. Field evaluation of 2 collar-mounted activity meters for detecting cows in estrus on a large pasture-grazed dairy farm. J. Dairy Sci. 95:3045-3056

Käppel, N. D., F. Proll, and G. Gauglitz. 2007. Development of a TIRF-based biosensor for sensitive detection of progesterone in bovine milk. Biosens. Bioelectron. 22:2295-2300.

Lane, E. A., M. A. Crowe, M. E. Beltman, and S. J. More. 2013. The influence of cow and management factors on reproductive performance of Irish seasonal calving dairy cows. Anim. Reprod. Sci. 141:34-41.

Löf, E., H. Gustafsson, and U. Emanuelson. 2014. Factors influencing the chance of cows being pregnant 30 days after the herd voluntary waiting period. J. Dairy Sci. 97:2071-2080.

Moons, K. G. M., D. G. Altman, J. B. Reitsma, J. P. A. Ioannidis, P. Macaskill, E. W. Steyerberg, A. J. Vickers, D. F. Ransohoff, and G. S. Collins. 2015. Transparent Reporting of a multivariable prediction model for Individual Prognosis or Diagnosis (TRIPOD): Explanation and elaboration. Ann. Intern. Med. 162:W1-W73.

Norman, H. D., J. L. Hutchison, and R. H. Miller. 2010. Use of sexed semen and its effect on conception rate, calf sex, dystocia, and stillbirth of Holsteins in the United States. J. Dairy Sci. 93:3880-3890.

Olynk, N. J., and C. A. Wolf. 2009. Stochastic economic analysis of dairy cattle artificial insemination reproductive management programs. J. Dairy Sci. 92:1290-1299.

Østergaard, S., N. C. Friggens, and M. G. G. Chagunda. 2005. Technical and economic effects of an inline progesterone indicator in a dairy herd estimated by stochastic simulation. Theriogenology 64:819-843.

R Development Core Team. 2008. R: A Language and Environment for Statistical Computing. R Foundation for Statistical Computing, Vienna, Austria.

Roche, J. R., N. C. Friggens, J. K. Kay, M. W. Fisher, K. J. Stafford, and D. P. Berry. 2009. Invited review: Body condition score and its association with dairy cow productivity, health, and welfare. J. Dairy Sci. 92:5769-5801.

Rook, A. J., J. France, and M. S. Dhanoa. 1993. On the mathematical description of lactation curves. J. Agric. Sci. 121:97-102. 
Rutten, C. J., W. Steeneveld, C. Inchaisri, and H. Hogeveen. 2014. An ex ante analysis on the use of activity meters for automated estrus detection: To invest or not to invest? J. Dairy Sci. 97:6869-6887.

Rutten, C. J., A. G. J. Velthuis, W. Steeneveld, and H. Hogeveen 2013. Invited review: Sensors to support health management on dairy farms. J. Dairy Sci. 96:1928-1952.

Schefers, J. M., K. A. Weigel, C. L. Rawson, N. R. Zwald, and N. B. Cook. 2010. Management practices associated with conception rate and service rate of lactating Holstein cows in large, commercial dairy herds. J. Dairy Sci. 93:1459-1467.

Schneider, M. P., E. Strandberg, U. Emanuelson, K. Grandinson, and A. Roth. 2007. The effect of veterinary-treated clinical mastitis and pregnancy status on culling in Swedish dairy cows. Prev. Vet. Med. 80:179-192.

Shahinfar, S., J. N. Guenther, C. D. Page, A. S. Kalantari, V. E. Cabrera, P. M. Fricke, and K. A. Weigel. 2015. Optimization of reproductive management programs using lift chart analysis and cost-sensitive evaluation of classification errors. J. Dairy Sci. 98:3717-3728.

Shahinfar, S., D. Page, J. Guenther, V. Cabrera, P. Fricke, and K. Weigel. 2014. Prediction of insemination outcomes in Holstein dairy cattle using alternative machine learning algorithms. J. Dairy Sci. 97:731-742.

Steeneveld, W., and H. Hogeveen. 2012. Economic consequences of immediate or delayed insemination of a cow in oestrus. Vet. Rec. 171:17.

Steyerberg, E. W., F. E. Harrell, G. Borsboom, M. J. C. Eijkemans, Y. Vergouwe, and J. D. F. Habbema. 2001. Internal validation of predictive models: Efficiency of some procedures for logistic regression analysis. J. Clin. Epidemiol. 54:774-781.

Tenhagen, B. A., M. Drillich, R. Surholt, and W. Heuwieser. 2004. Comparison of timed AI after synchronized ovulation to AI at estrus: Reproductive and economic considerations. J. Dairy Sci. 87:85-94.

Val-Arreola, D., E. Kebreab, J. Dijkstra, and J. France. 2004. Study of the lactation curve in dairy cattle on farms in central Mexico. J. Dairy Sci. 87:3789-3799.

van der Drift, S. G. A., R. Jorritsma, J. T. Schonewille, H. M. Knijn, and J. A. Stegeman. 2012. Routine detection of hyperketonemia in dairy cows using Fourier transform infrared spectroscopy analysis of beta-hydroxybutyrate and acetone in milk in combination with test-day information. J. Dairy Sci. 95:4886-4898.

Wickham, H. 2007. Reshaping data with the reshape package. J. Stat. Softw. 21:1-20.

Wickham, H., and R. Francois. 2014. dplyr: A grammar of data manipulation. Accessed May 18, 2016. https://github.com/hadley/dplyr.

Wilmink, J. B. M. 1987. Adjustment of test-day milk, fat and protein yield for age, season and stage of lactation. Livest. Prod. Sci. $16: 335-348$

Windig, J. J., M. P. L. Calus, and R. F. Veerkamp. 2005. Influence of herd environment on health and fertility and their relationship with milk production. J. Dairy Sci. 88:335-347.

Wood, P. D. P. 1967. Algebraic model of lactation curve in cattle. Nature 216:164-165. 